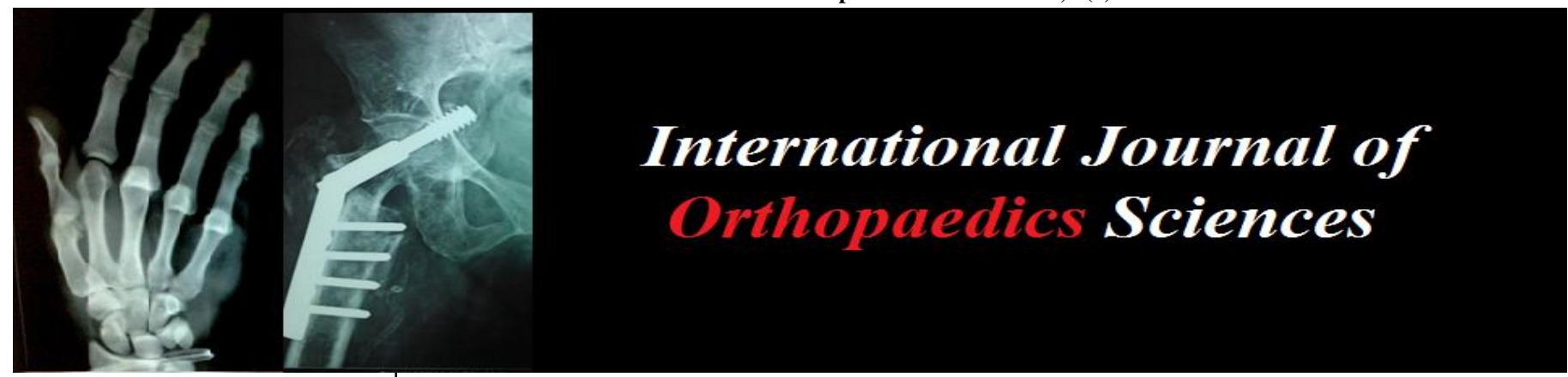

ISSN: $2395-1958$

IJOS 2019; 5(3): 391-393

(C) 2019 IJOS

www.orthopaper.com

Received: 08-05-2019

Accepted: 12-06-2019

Dr. Umang Agrawal MS

Assistant Professor, Department of Orthopaedics, Shah Medical

College Campus, Jamnagar,

Gujarat, India
Correspondence

Dr. Umang Agrawal MS

Assistant Professor, Department

of Orthopaedics, Shah Medical

College Campus, Jamnagar,

Gujarat, India

\section{Efficacy of pregabalin in management of postoperative pain and functional outcomes in immediate postoperative period following total knee replacement}

\section{Dr. Umang Agrawal MS}

DOI: https://doi.org/10.22271/ortho.2019.v5.13g.1559

\begin{abstract}
Introduction: Immediate postoperative pain after total knee arthroplasty has remained a serious problem, which prolongs the length of hospital stay and functional recovery. Thus multimodal pain management protocol are followed in these patients. Our study aims to assess the efficacy of pregabalin use in pain management and functional recovery of patients in the immediate postoperative period.

Materials and Methods: We did a prospective randomised case control study by taking patients operated for total knee arthroplasty and receiving multimodal analgesia. We divided them into two groups with 100 patients in each group. GROUP A (with pregabalin) AND GROUP B (without pregabalin). We have given Pregabalin in recommended and FDA approved safe dosage of $75 \mathrm{mg}$ one tab bedtime for a period of three weeks postoperatively along with selective COX-II inhibitors NSAIDs, (celecoxib) for three weeks. In another group, we have omitted the use of pregabalin and gave only celecoxib for three weeks. Patients were assessed by knee society score, WOMAC score, and VAS scores at $24 \mathrm{hr}$ after surgery, $48 \mathrm{hr}$ after surgery, at two weeks.

Results: The patients were divided into two groups each having 100 patients and were compared using statistical tools using one way ANOVA Test, The Vas Score, WOMAC Score, knee Range of motion and Knee Society Score showed statistically significant change in pregabalin group.

Discussion: We are performing this comparative study to assess its role in pain management and also its effects on functional outcomes of the patient. Few literature suggests that Pregabalin is effective for reducing opioid consumption after primary TKA and reducing respiratory, renal, and hemodynamic complications.

Conclusion: In my study the use of Pregabalin is suggestive of improvement in pain score and functional outcomes in the patients in immediate post-op period over 3 months. However, future studies regarding doses and pregabalin medication are required.
\end{abstract}

Keywords: Total knee arthroplasty, pregabalin, pain management

\section{Introduction}

Total knee replacement is the well-established treatment for the osteoarthritis of the knee. Postoperative pain after total knee arthroplasty has remained a serious problem, which prolongs the length of hospital stay and functional recovery. Therefore, appropriate pain management protocol is necessary to relieve postoperative pain and achieve early functional recovery. Thus multimodal pain management protocol that includes the use of regional anaesthesia, peripheral nerve blocks (adductor canal blocks), periarticular injections, longacting bupivacaine, Opiods ${ }^{[1]}$, nonsteroidal anti-inflammatory drugs, including selective cyclooxygenase inhibitors and gabapentinoids are followed in these patients. Pregabalin (LYRICA) provides pain control by binding to the $\alpha 2 \delta$ subunit of the voltage-gated calcium channel to reduce the calcium influx into the terminal of presynaptic and thus can relieve the sensitization of peripheral nociceptive nerve terminals and central neurons ${ }^{[2]}$. The Pregabalin is used in most of the multimodal pain management protocol of total knee replacement in the postoperative period. We are performing this study to assess the efficacy of Pregabalin in pain management and its overall effect on patient functional outcomes. 


\section{Aim and objectives}

Our study aims to assess the efficacy of pregabalin use in pain management and functional recovery of patients in the immediate postoperative period as adequate pain control allows patients to achieve accelerated recovery by improving rehabilitation and quality of life.

\section{Inclusion criteria}

1. All patients operated by total knee replacement receiving multimodal analgesia.

2. Both males and females were taken in the study.

\section{Exclusion criteria}

1. Revision cases of total knee replacement

2. Patients allergic to Pregabalin

3. ASA physical status of IV

4. History of neuropathic pain or any other chronic pain condition, other than osteoarthritis pain

5. Currently enrolled in another investigational study.

\section{Materials and Methods}

Type of study: prospective randomized case-control study

Total no. of patients: 200

After ethical clearance and informed consent we have taken patients operated by total knee replacement and receiving multimodal pain management at Sunshine Hospital, secunderabad. The patients were given multimodal analgesia in the form of spinal block, adductor canal block, periarticular injection while surgery and selective COX-II Inhibitors and Pregabalin postoperatively. The patient was divided into two groups (100 each) with both receiving multimodal analgesia -

1. With Pregabalin (GROUP A)

2. Without Pregabalin. (GROUP B)

We have given Pregabalin in recommended and FDA approved safe dosage of $75 \mathrm{mg}$ one tab bedtime for a period of three weeks postoperatively along with selective COX-II inhibitors NSAIDs, (celecoxib) for three weeks. In another group, we have omitted the use of pregabalin and gave only celecoxib for three weeks.

Patients were assessed by knee society score, WOMAC score, and VAS scores at $24 \mathrm{hr}$ after surgery, $48 \mathrm{hr}$ after surgery, at two weeks, at six weeks and at 12 weeks. The pain was measured using the visual analog scale (VAS), with 0 denoting no pain and 10 indicating the greatest pain they experienced. Both groups were compared regarding the results obtained from our scores by proper statistical analysis using t-test and ANOVA. The patients were also observed for any side effects developing due to the use of the pregabalin like diplopia, blurry vision/blurriness, nausea, dizziness, drowsiness, sleepiness, somnolence, dry mouth, edema, swelling, palpitations, giddiness, and difficulty concentrating. The patients developing any side effects were omitted with the drug and excluded from the study.

\section{Results}

The patients were divided into two groups each having 100 patients and were compared using statistical tools using one way ANOVA Test

\section{Knee society pain Score}

1. The p-value is less than 0.001 ; hence we can conclude that there is a significant change in the KS score with Pregabalin

\section{WOMAC Score}

1. The p-value is less than 0.001 ; hence we can conclude that there is a significant change in the WOMAC score with Pregabalin

2. Based on the Tukey's post hoc test, the p-value between $24 \mathrm{hrs}$ and $48 \mathrm{hrs}$ is $0.069>0.05$, hence we can conclude that there is no significant change between $24 \mathrm{hrs}$ and 48 hrs WOMAC score.

\section{Knee Rom}

1. The $\mathrm{p}$-value is less than 0.001 ; hence we can conclude that there is a significant change in the Knee Rom score with PREGABALIN

2. Based on the Tukey's post hoc test, the p-value between $24 \mathrm{hrs}$ and $48 \mathrm{hrs}$ is $0.943>0.05$, hence we can conclude that there is no significant change between $24 \mathrm{hrs}$ and 48 hrs WOMAC score.

\section{VAS Score}

1. The p-value is less than 0.001 ; hence we can conclude that there is a significant change in the VAS score with Pregabalin

\section{WOMAC functional score}

1. The p-value is less than 0.001 , hence we can conclude that there is a significant change in the Womac Functional score with PREGABALIN

2. Based on the Tukey's post hoc test, the p-value between $24 \mathrm{hrs}$ and $48 \mathrm{hrs}$ is $0.069>0.05$, hence we can conclude that there is no significant change between $24 \mathrm{hrs}$ and 48 hrs Womac Functional score.

\section{Knee society score}

1. The p-value is less than 0.001 ; hence we can conclude that there is a significant change in the Knee Society score with Pregabalin

2. Based on the Tukey's post hoc test, the p-value between $24 \mathrm{hrs}$ and $48 \mathrm{hrs}$ is $0.208>0.05$, hence we can conclude that there is no significant change between $24 \mathrm{hrs}$ and 48 hrs Knee Society score.

\section{Discussion}

Total knee arthroplasty (TKA/TKR) surgeries have increased over the past decade and postoperative pain management is essential to enhance recovery and allow patient to follow rehabilitation protocol easily. Thus multimodal analgesia seems to reduce pain by different molecules acting over different receptors reducing nociceptive sensation. We have seen studies regarding the role of Pregabalin in pain relief along with the multimodal analgesia ${ }^{[3]}$. Most of them suggested positive efficacy. Few studies nullify their role. We are performing this comparative study to assess its role in pain management and also its effects on functional outcomes of the patient. Few literatures suggests that Pregabalin is effective for reducing opioid consumption after primary TKA and reducing respiratory, renal, and hemodynamic complications ${ }^{[4]}$. Some literatures suggests that pregabalin could improve pain control at 24 and $48 \mathrm{~h}$ with rest, reduce morphine consumption, and improve the knee flexion degree as well as decrease the incident rate of nausea, vomiting, and pruritus and increasing the incident rate of dizziness after TKA ${ }^{[5]}$. However, future studies regarding doses and pregabalin medication are required. Thus we are performing this study to address our question of the role of Pregabalin in pain 
management and functional outcomes of postoperative TKR patients. Few studies suggest that there was no evidence that the use of PREGABALIN improved short or long term function, with no difference in knee flexion at 48 hours incidence of chronic pain at 3or 6 months. There were associated with a reduction in the incidence of nausea but Pregabalin was associated with a clinically relevant increase in sedation.

\section{Limitations of Study}

1. This study only studied the effects of oral Pregabalin on pain control after TKA and did not investigate its applicability as an injectable medication.

2. We gave only $75 \mathrm{mg}$ of pregabalin in this study, and the effects of a higher dose of pregabalin $(150 \mathrm{mg}$ or $300 \mathrm{mg}$ ) were not studied.

3. This study included small sample size, and greater effects may have been noted with a larger patient population. Additionally, with a larger patient population, a greater number of side effects may have been detected.

\section{Conclusion}

The study compared the efficacy of the use of pregabalin in TKR patients post-surgery. Thus at first and second post-op day the results are statistically better in pregabalin group. In my study the use of Pregabalin is suggestive of improvement in pain score and functional outcomes in the patients in immediate post-op period over 3months. However, future studies regarding doses and pregabalin medication are required.

\section{Reference}

1. Benyamin R, Trescot AM, Datta $\mathrm{S}$ et al. Opioid complications and side effects. Pain Physician. 2008; 11(2):S105-S120.

2. Grosu I, Lavand'homme P, Thienpont E. Pain after knee arthroplasty: an unresolved issue. Knee Surg. Sports Tramatol Arthrosc. In press.

3. Goyal N, McKenzie J, Sharkey PF, Parvizi J, Hozack WJ, Austin MS. The 2012 Chitranjan Ranawat award: intraarticular analgesia after TKA reduces pain: a randomized, double-blinded, placebo-controlled, prospective study. Clin Orthop Relat Res. 2013; 471(1):64-75.

4. Dorr LD, Raya J, Long WT, Boutary M, Sirianni LE. Multimodal analgesia without parenteral narcotics for total knee arthroplasty. J Arthroplasty. 2008; 23(4):502508 .

5. $\mathrm{Ng} \mathrm{FY,} \mathrm{Ng} \mathrm{JK}$, Chiu $\mathrm{KY}$, Yan $\mathrm{CH}$, Chan $\mathrm{CW}$. Multimodal Periarticular injection VS continuous femoral nerve block after total knee arthroplasty: a prospective, crossover, randomized clinical trial. J Arthroplasty. 2012; 27(6):1234-1238.

6. Chelly JE, Greger J, Gebhard R et al. Continuous femoral blocks improve recovery and outcome of patients undergoing total knee arthroplasty. J Arthroplasty. 2001; 16(4):436-445

7. Kelley TC, Adams MJ, Mulliken BD, Dalury DF. Efficacy of multimodal perioperative analgesia protocol with periarticular medication injection in total knee arthroplasty: a randomized, double-blinded study. J Arthroplasty. 2013; 28(8):1274-1277. 